Article

\title{
Experimental and Numerical Analysis of Deformation in a Rotating RC Helicopter Blade
}

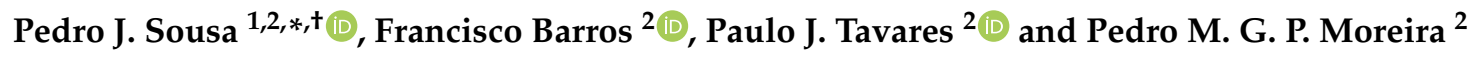 \\ 1 Faculty of Engineering, University of Porto, 4200-465 Porto, Portugal \\ 2 INEGI-Institute of Science and Innovation in Mechanical and Industrial Engineering, \\ 4200-465 Porto, Portugal; fbarros@inegi.up.pt (F.B.); ptavares@inegi.up.pt (P.J.T.); \\ pmoreira@inegi.up.pt (P.M.G.P.M.) \\ * Correspondence: ppsousa@fe.up.pt \\ † Current address: Rua Dr. Roberto Frias, 400, 4200-465 Porto, Portugal.
}

Received: 13 May 2020; Accepted: 28 June 2020; Published: 2 July 2020

check for updates

\begin{abstract}
Rotating structures are important and commonly used in the transportation and energy generation fields, where a better understanding of the deformations these structures endure is essential for both the design and maintenance phases. This work presents a novel image sensing methodology for measuring the displacements of rotating parts in operation due to dynamic loading. This methodology employs 3D digital image correlation combined with a custom stroboscopic lighting solution to achieve apparent stillness of the target while it rotates and then processes the acquired data to remove small imprecisions and align it to the rotor's intrinsic coordinate system. It was applied to an RC helicopter, whose blade deformation was measured and compared with a computational model, using fluid-structure interaction between computational fluid dynamics (CFD) and finite element analysis (FEA). Using live measurement techniques, it was possible to obtain the actual behaviour of the blades, which can be used to validate and tune computational models. The proposed methodology complements the methods available in the literature, which were centred around relative out-of-plane displacements, by enabling the comparison of absolute out-of-plane and in-plane ones.
\end{abstract}

Keywords: digital image correlation; computational fluid dynamics; fluid-structure interaction; rotating structures; structural monitoring; structural integrity; dynamic loading

\section{Introduction}

Rotating structures are widespread and important components in fields such as transportation and energy generation, where they are usually part of important machines, such as gas and wind turbines [1]. In many of these cases, the employed rotating structures are long and slender, causing them to exhibit large deformations in operation $[2,3]$. Thus, it is important to be able to predict and measure their displacements, in order to be able to understand their behaviour and improve the efficiency of the systems to which they belong.

In recent years, this type of structure has been analysed using numeric or experimental approaches. For example, Bazilevs et al. [3] and Santo et al. [4] present numeric approaches that employ fluid-structure interaction to analyse the blades' displacements in wind turbine rotors, with the aim of predicting the output power of the turbine. Additionally, some other works, without fluid-structure interaction, are also present in the literature. Among these, Stürmer et al., when working with counter-rotating open rotor (CROR) propulsion systems [5], reported that the velocity distributions obtained using particle image velocimetry (PIV) and computational fluid dynamics analysis (CFD) show differences, where the latter overestimates the velocity magnitudes in the rotor slipstream 
and presents discrepancies in the vorticity field. These differences were attributed to the lack of fluid-structure interaction in their CFD analysis and the fact that there is deformation of the blades, changing the operating point away from the nominal values by, for example, altering the effective blade pitch. Stürmer et al. also note that the availability of blade deformation data can improve the quality of numerical models of these systems [5], as well as the usage of fluid-structure interaction.

Experimental work on the analysis of similar wind turbines has also been undertaken in the literature. For example, Winstroth et al. [2,6,7] employed digital image correlation (DIC) to measure blade displacements in multi-megawatt wind turbines. Besides this, similar approaches have also been applied to displacement monitoring of other related rotating objects, such as helicopters [8] and turboprop airplanes [9].

However, experimental validation of simulations centring on structural behaviour is scarce in the literature. The work developed by Winstroth et al. [6] is one of those few cases, where the blade displacements for a wind turbine were measured using their DIC-based methodology [2,7] and compared to an aeroelastic simulation. That work involved the comparison of relative out-of-plane deflections and obtained good agreement. However, they were unable to compare absolute out-of-plane deformations nor any in-plane deformations [6].

The current project is part of a program for monitoring response change in turbine blades along their lifespan with contactless, image-based sensing. This work on fluid-structure interaction on the modelling side, as well as future modal analysis and vibration monitoring work on the experimental side, will complete this program that started with a focus on deformation monitoring alone.

The central goal of the reported work is thus to analyse the deformation of the rotating blades of an RC helicopter using experimental and numerical approaches, comparing the obtained absolute out-of-plane and in-plane displacements. The first part is based on digital image correlation applied to rotating objects as described in [10-12]. For the latter, the numerical results are obtained using coupled fluid-structure interaction between computational fluid dynamics and finite element analysis models.

The developed measurement methodology can be used for different rotation speeds and geometries, with potential usage in wind turbines, helicopters and airplanes. Among these, it can be used as a complement for numerical models. For simulations using fluid-structure interaction, this methodology can be used to validate the obtained displacement field by comparing with real measurements. On the other hand, for simulations using a simplified model without fluid-structure interaction, it would be possible to introduce the deformed shape in order to improve the results with smaller computational cost.

The present work follows a previous one [13] by the authors, where significant discrepancies were found between the experimental and numeric results, which were resolved here. It is organised as follows: in Section 2, the experimental approach is described, where the operation of the main components of the setup is explained; in Section 3, the numerical model is described, defining the problem starting from shape acquisition and material properties; in Section 4, the results that were obtained from the two approaches are compared; and in Section 5 conclusions are presented.

\section{Experimental Approach}

The setup used in this work is shown in Figure 1. Point-Grey Gazelle industrial cameras (4.1 MP, $150 \mathrm{fps}$ ) and a custom illumination system, developed in [14], were used to acquire images of the RC helicopter's rotating blades at $680 \mathrm{rpm}$.

The experimental data were obtained applying the methodology described in [10-12], following the logic of the schematic shown in Figure 2.

A trigger signal is generated from the rotation of the blades using a photodiode, which will detect the interruption of a laser beam by a blade. This signal is then processed by the trigger controller, which will control both the exposure of the cameras and the light pulse. 


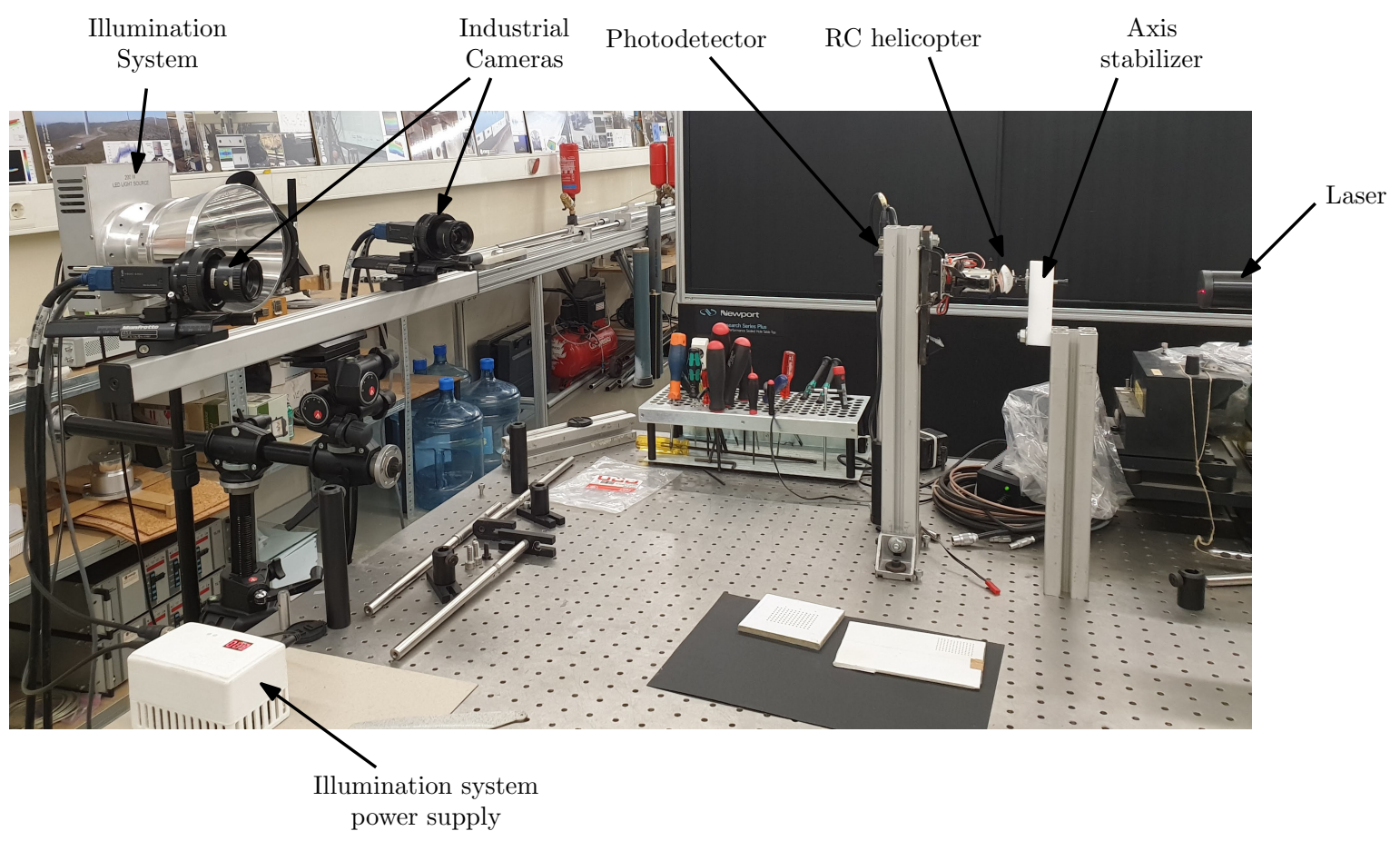

Figure 1. Experimental setup for the new image sensing methodology.

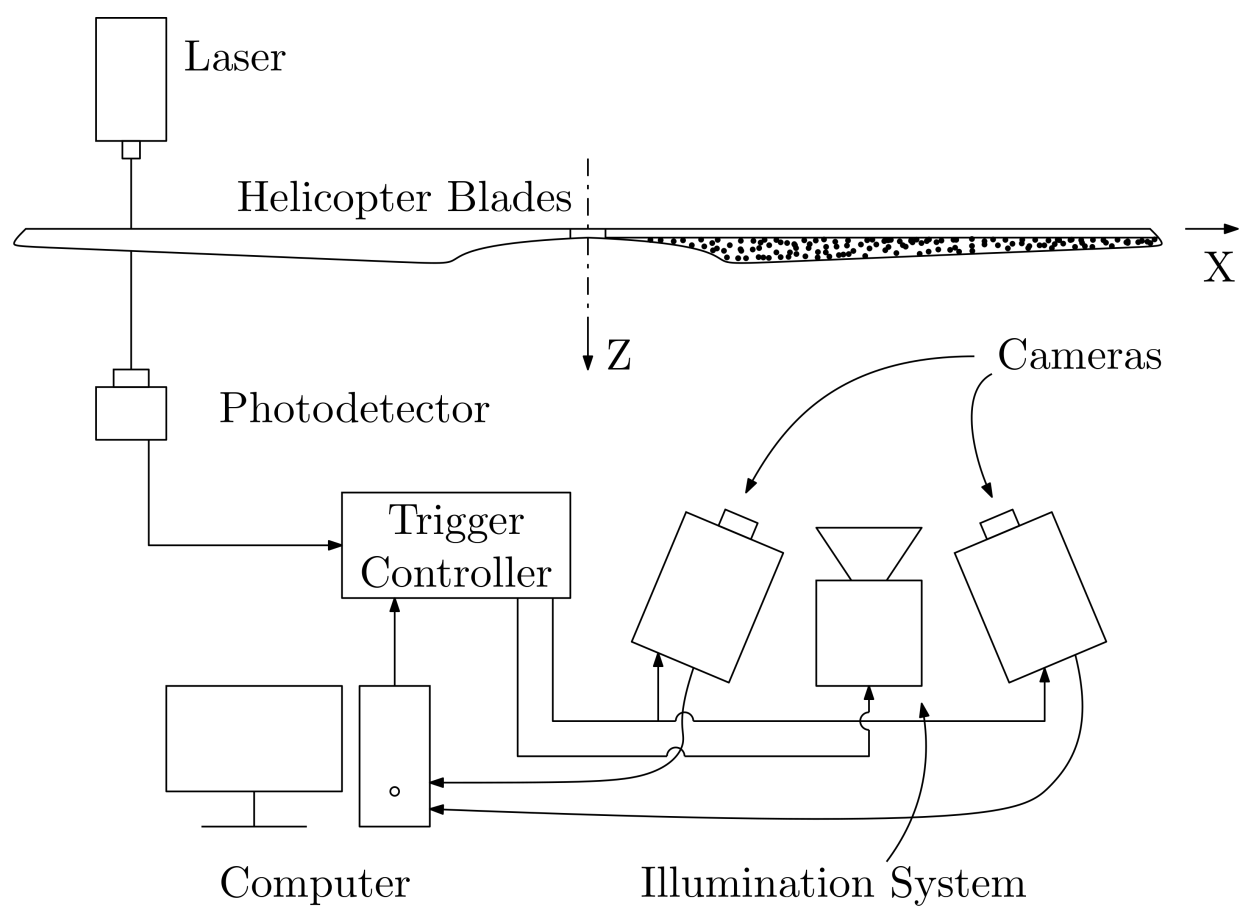

Figure 2. Schematic of the experimental setup employed for the new image sensing methodology.

The central part of this setup, the trigger controller system shown in Figure 3, is built around a dsPIC33EV256GM102 microcontroller from Microchip Technology Inc (Chandler, AZ, USA). 


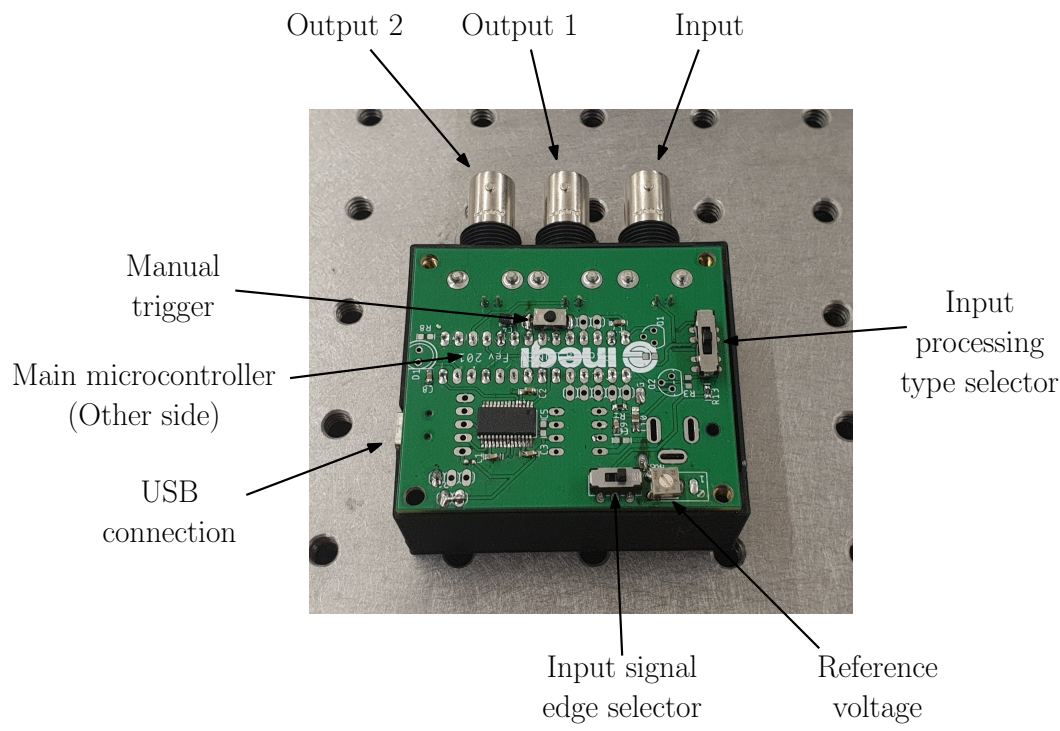

Figure 3. Photo of the developed trigger controller.

As previously mentioned, the main functionality of this system is to receive periodic signals from the rotating objects ( $n$ pulses per rotation) and, after counting a defined number of pulses $(m)$, generate two independent trigger signals, for use with cameras and/or illumination systems.

If the selected count is different from the total number of pulses per rotation $(m \neq n)$ and it is also not a multiple, the obtained images will be from a different blade in each cycle. This functionality can be used to, for example, inspect multiple blades or limit the amount of data that are generated.

The system architecture is described in Figure 4. The input signal is processed using external circuitry in order to obtain a $5 \mathrm{~V}$ signal with active rising edge. There are two types of processing, with both inverting and non-inverting options: one transistor-based and another comparator-based. The first is faster but requires input voltages above $5 \mathrm{~V}$, while the second one is more versatile but requires the definition of a reference voltage. The resulting signal is then processed using only hardware modules to ensure high repeatability in the system response.

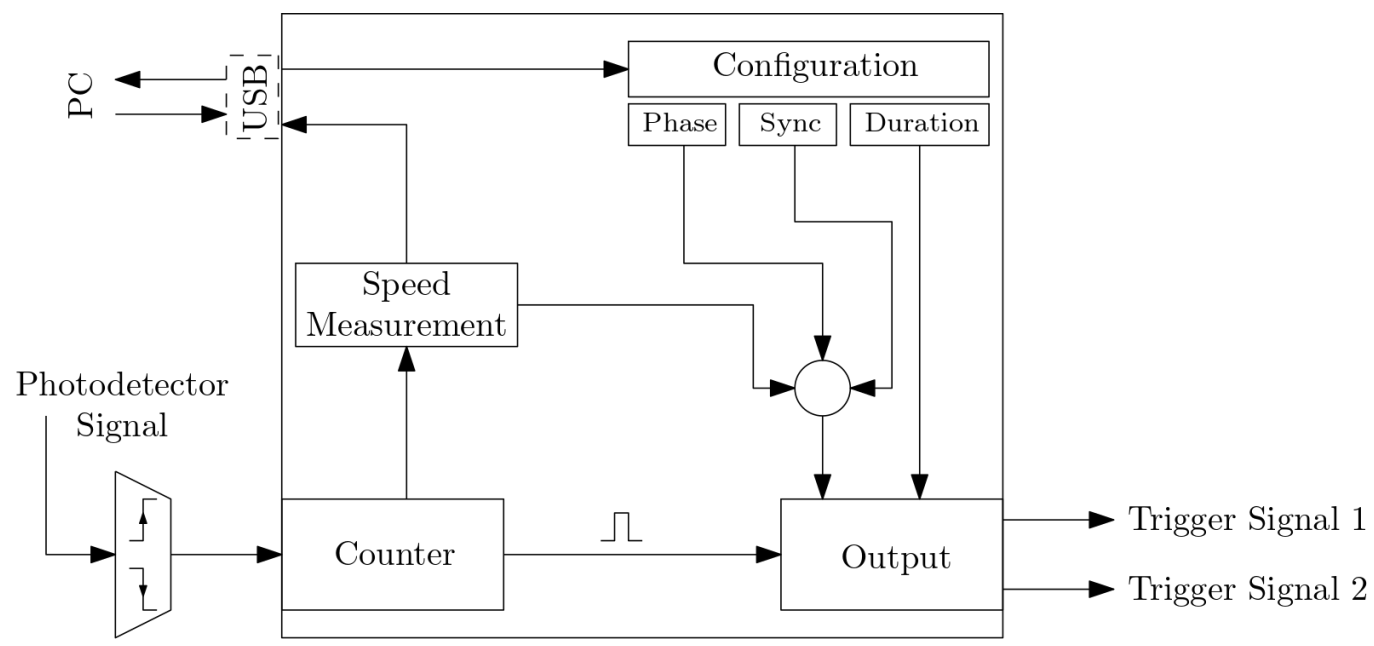

Figure 4. Operation logic for the trigger system.

This processed input signal is fed directly into an asynchronous counter, that will count up to $m$ pulses. As soon as that value is reached, it will trigger the output compare module, which outputs two independent signals. 
In parallel, the time between successive output pulses is measured using a Timer module and the object's rotation speed is calculated. Using this, it is possible to pause the object in different angular positions, by changing the trigger delay to include a phase component.

Using the USB connection, it is possible to register the measured speed values and define some user-configurable parameters, which include the input pulse count $(m)$, the number of pulses per rotation $(n)$, the phase $(\Phi)$ and each output signal's parameters (sync and duration).

These output pulses are characterised by the total delay and duration. The first is obtained from the user-defined sync delay and phase angle, while the second is directly defined by the user. The phase angle is converted to a delay by considering the latest speed measurement. Figure 5 shows the relation between an output pulse, its configuration and the input. Reconfiguration is always performed after an output pulse, where the next pulse is redefined to reflect the newly measured rotation speed and any configuration changes that the user may have made in the meantime.

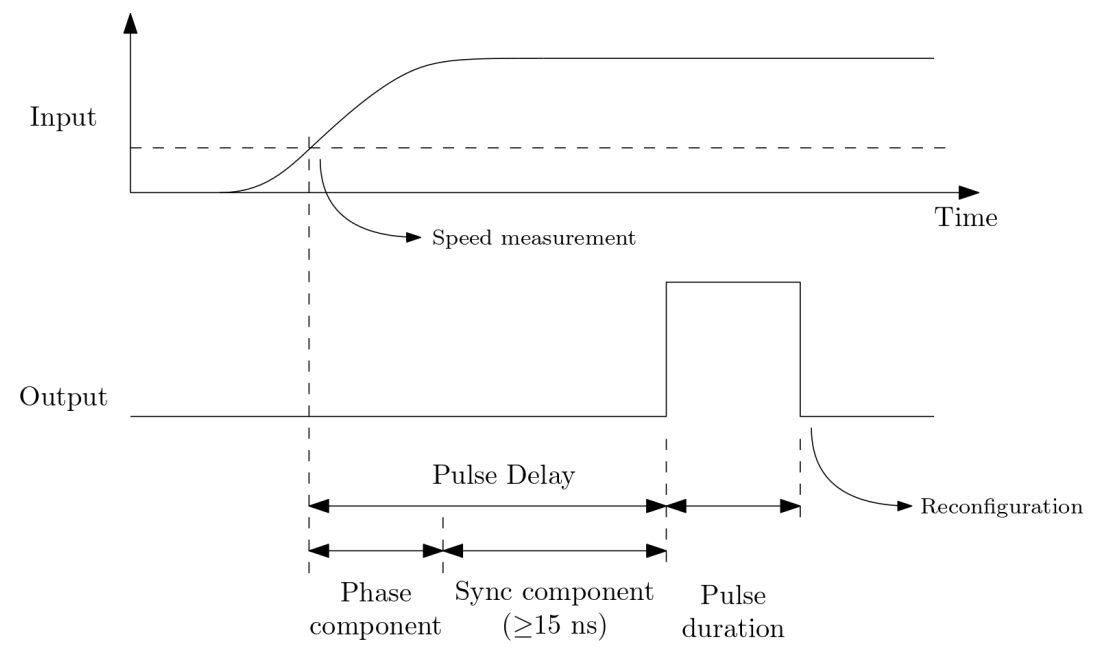

Figure 5. Components and timing of the output pulses.

In order for this controller to react to the interruption of a laser beam, it must be interfaced with a detector. For this purpose, a custom photodetector system was developed around a OSD15-0 photodiode from OSI Optoelectronics (Hawthorne, CA, USA). The main reason for this development was the need for a cheap solution for a fast photodetector with larger active area than previously used solutions $[10,11]$, as their small active area would cause difficulties in the setup phase of the experiment, requiring precise alignment. Additionally, the development of a custom solution allows more leeway for adjustment if necessary.

There are two main modes of operation for photodiodes, photoconductive and photovoltaic [15]. In the first mode, an external reverse bias is applied to the photodiode, i.e., a negative voltage is applied to the anode, obtaining a current through the circuit as a function of the incoming light. This mode has several advantages, such as increased speed and a very linear response, as well as some drawbacks, such as a higher dark current which also increases the noise [15-17].

On the other hand, the photovoltaic mode, traditionally used in solar cells, is obtained by zero biasing the photodiode, i.e., the anode is connected to ground. It has the main advantage of reduced dark current and its associated lower noise, but also a significant drawback in its non-linearity [15-17].

As the employed laser beam has considerable power, the most important parameter here is the response speed, at the expense of sensitivity and noise. As such, the photoconductive mode was employed.

The OSD15-0 belongs to a series of diodes specially developed for use in photoconductive mode. It features a rise time of $9 \mathrm{~ns}$ and a maximum bias voltage of $30 \mathrm{~V}$ with an active area of $15 \mathrm{~mm}^{2}$ (a $3.8 \times 3.8 \mathrm{~mm}$ square), fifteen times larger than the previous solution [17]. 
The diode's signal was amplified using the circuit of Figure 6, which is centred around the THS4631 Wideband Operational Amplifier from Texas Instruments (Dallas, TX, USA). This device was selected due to its bandwidth, a fast slew rate and support for a supply voltage of $10 \mathrm{~V}$, which was selected as a compatibility feature, in order to have a similar output range as the photodetectors used in previous experiments.

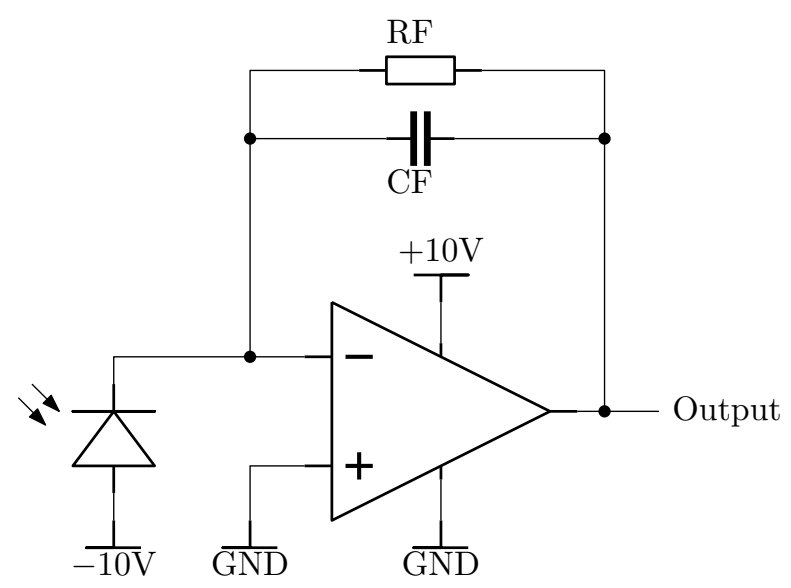

Figure 6. Transimpedance amplification of the photodiodes' signal in the laser-based approach.

The feedback resistor and capacitor $\left(R_{F}\right.$ and $\left.C_{F}\right)$ were calculated following the datasheet of the amplifier [18], obtaining values of $9 \mathrm{pF}$ and $2200 \Omega$ respectively.

The final result is shown in Figure 7 and it is described in Figure 8.

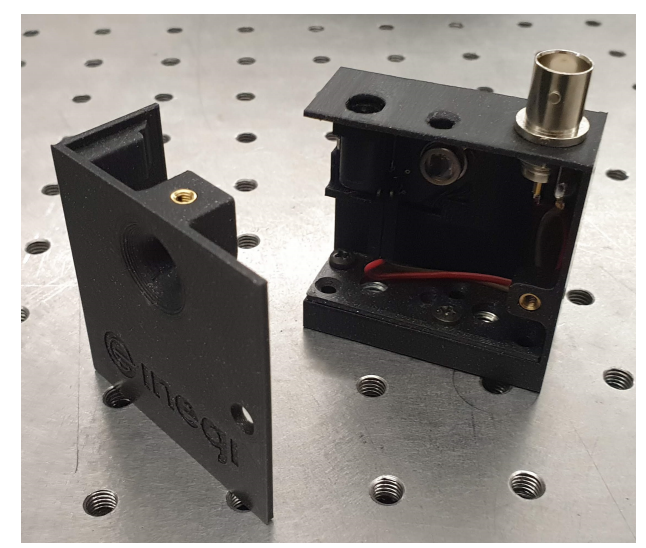

Figure 7. Custom photodetectors for laser-based triggering.

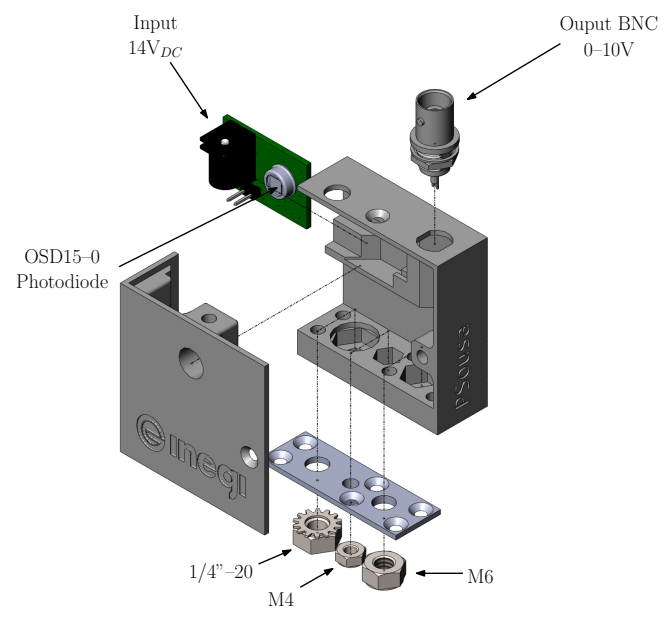

Figure 8. Main components and features of the developed OSD15-0-based photodetector system. 


\section{Numerical Model}

In this section, a coupled CFD-FEA numerical model will be developed as a comparison point for the experimental measurements. Thus, it is of utmost importance to accurately represent the physical situation in the model. With this in mind, the shape of the blades and their material properties were obtained.

\subsection{Shape Acquisition}

For the first approaches, a single acquisition using a commercial structured light scanner, a DAVID SLS-2 from DAVID Vision Systems (Koblenz, Germany) (Figure 9), was used to create the 3D model for simulation. This however resulted in a poor quality model, contributing to the considerable deviation between the numeric results and the experimental ones [13]. The reason for this was not only the assumption of constant thickness, but also the loss of a few millimetres near the edges and inconsistent shape due to reflections.

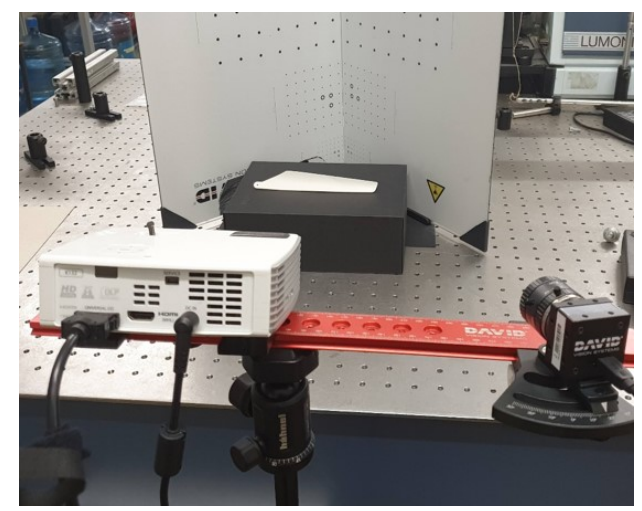

Figure 9. Shape acquisition setup using a DAVID Structured Light 3D Scanner.

In order to avoid these shape inconsistencies, a coating of dry powder can be added to the target object, reducing reflections and obtaining a controlled acquisition environment. The effect of this change can be clearly seen in Figure 10, where the acquired blade root shape is shown, without dry powder on the left and with it on the right. The presence of phantom peaks and valleys on the first one is very noticeable and is representative of the effect reflections have on this type of acquisition.

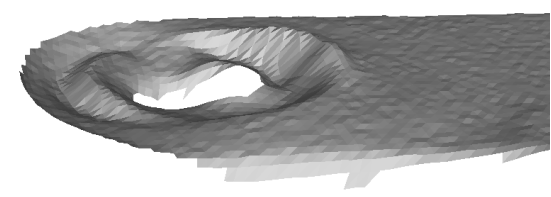

(a)

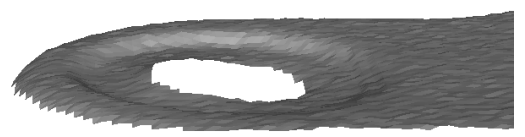

(b)

Figure 10. The effects of reflection in the structured light shape acquisition procedure. (a) Without dry powder reflections cause geometry errors. (b) With dry powder the obtained surface correctly matches the geometry.

On the other hand, the remaining two problems, namely constant thickness and edge misrepresentation, can be solved at once by performing and combining multiple acquisitions, from different sources and points of view. In Figure 11, all the used sources are represented. Some of 
the surfaces acquired using the previously mentioned DAVID SLS-2 are shown Figure 11a. The two shapes shown are one from the front of the blade and another from the back; nonetheless, surfaces from the sides were acquired using this scanner. Another method that was employed, whose result is shown in Figure 11b, is a custom laser line scanner, created using a simple laser and camera setup. Besides these two sources, the previous experimental applications of DIC are also able to supply shape information in the undeformed situation, shown in Figure 11c.

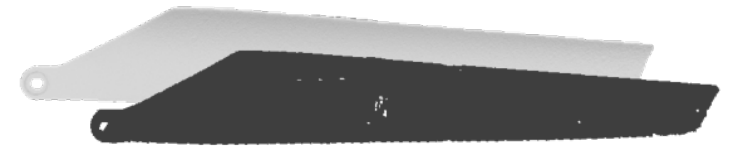

(a) Structured light scanner

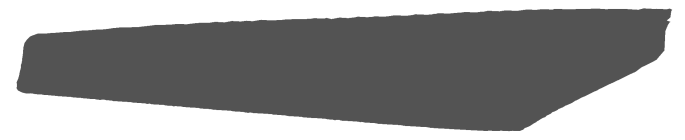

(b) Custom line laser scanner

(c) Digital image correlation results

Figure 11. The multiple sources employed for shape information.

All these data sources were combined in MeshLab (2016.12, Open-Source) [19]. The different surfaces were manually aligned between themselves, using overlapping regions as a reference, to obtain a surface-based model of the blade, Figure 12. This long process, however, resulted in an incomplete model with some holes, mainly along the edges of the blade.

Afterwards, this surface-model was imported into SolidWorks (2019, Dassault Systèmes, Vélizy-Villacoublay, France) and used as a guide for the manual definition of a parametric solid model, Figure 13, that was used for the simulation.

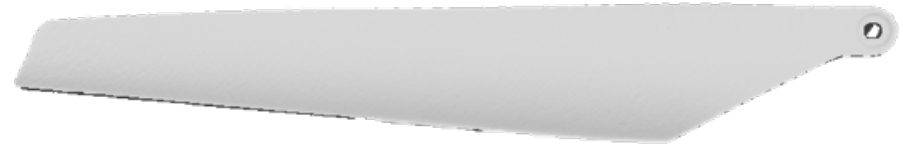

Figure 12. The obtained merged shape surface, created by matching the different sources.

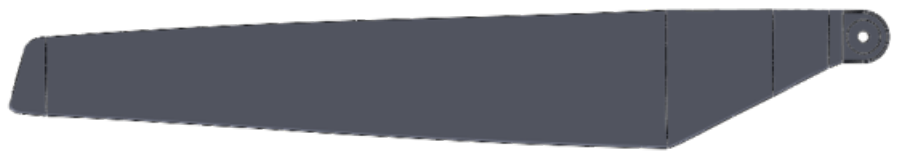

Figure 13. Final parametric model of the blade.

\subsection{Material Properties}

Having the 3D shape of the blades, it is then necessary to acquire the material properties: Young's modulus, Poisson's ratio and density.

There are a few materials that are commonly used to make RC helicopter blades, such as ABS (acrylonitrile butadiene styrene, a terpolymer made by polymerizing styrene and acrylonitrile in the presence of polybutadiene that is stronger than pure polystyrene). However, particular details for the one used in these is not made available by the manufacturer, as it is not necessary for its typical usage and consequently there are no tabled values which could be used. Thus, the only available approach is to obtain those parameters experimentally.

The easiest one, density, was approximated by dividing the measured weight of the blade by the 3D model's volume, obtaining a density of $1127 \mathrm{~kg} \mathrm{~m}^{-3}$. 
For the other two parameters, a specimen was cut from the region with the most constant cross-section of a blade and painted with a speckle pattern, in order to enable the use of DIC to measure both the transversal and longitudinal strain.

Afterwards, the specimen was subjected to a tensile test in an Instron E1000 machine (Instron, Norwood, MA, USA), Figure 14, until it reached a displacement of $1 \mathrm{~mm}$. In addition to the machine's $\log$ of load and displacement, a 2D digital image correlation system was used to provide directional displacements, which are mandatory to obtain the Poisson ratio, as it can be calculated as:

$$
v=-\frac{\varepsilon_{\text {transversal }}}{\varepsilon_{\text {axial }}}
$$

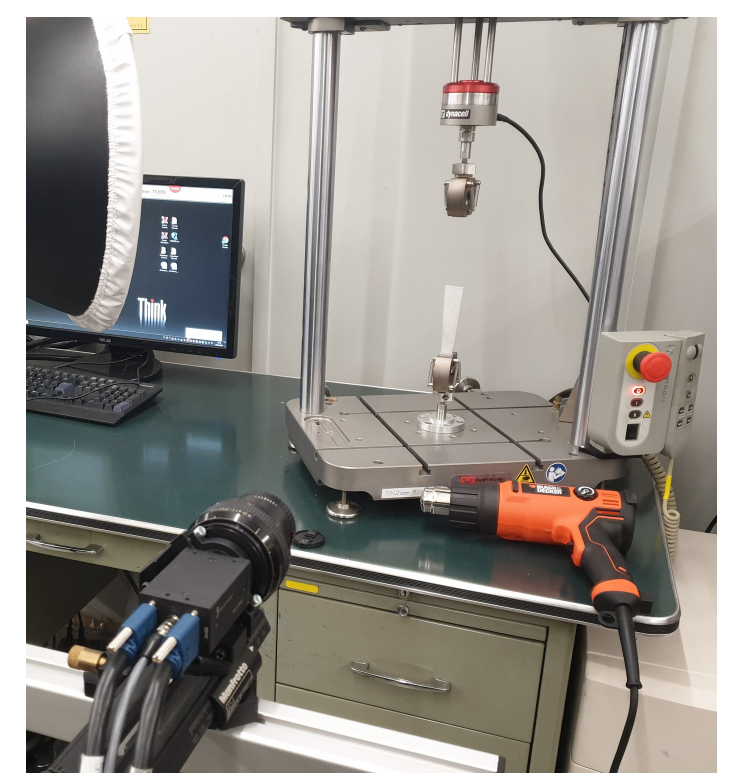

Figure 14. Setup used for the measurement of the blade's Young modulus and Poisson ratio.

Using the machine's outputs, it is possible to draw a force-displacement plot for the loading, Figure 15a. In order to obtain the Young's modulus from this information, Hooke's law $\left(E=\frac{\sigma}{\varepsilon}\right)$ was applied. Thus, it was necessary to convert the force-displacement curve to a stress-strain one. This can be done considering the section area of $A=11.5 \times 1.5=17.25 \mathrm{~mm}^{2}$ and the active length of $60 \mathrm{~mm}$, applying the equations $\sigma=\frac{F}{A}$ and $\varepsilon=\frac{\Delta L}{L}$ and obtaining the curve of Figure $15 \mathrm{~b}$.

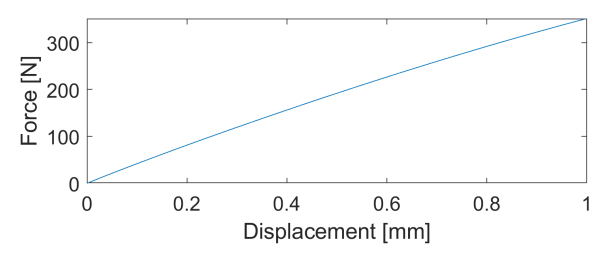

(a) Force-Displacement curve

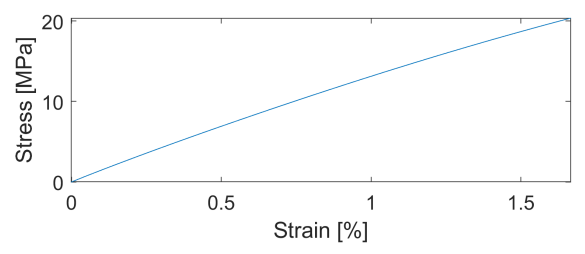

(b) Stress-Strain curve

Figure 15. Loading results from the Instrom test machine, resulting in an approximation of the blade's Young's modulus. 
Approximating the resulting stress-strain curve to a straight line, a value of $1221.72 \mathrm{MPa}$ is obtained for Young's modulus. For the simulation, this value was rounded to the hundreds of mega pascal, $1200 \mathrm{MPa}$, as there is still some uncertainty.

The same experiment was used to calculate the Poisson ratio of the material based on the DIC results, where the $X$ and $Y$ directions were aligned with the axial and transversal ones, thus calculating the ratio as:

$$
v=-\frac{\varepsilon_{x x}}{\varepsilon_{y y}}
$$

for each point of the image, obtaining the representation shown in Figure 16. It is possible to see some variations in the results. Thus, they were exported to MATLAB (R2018a, The MathWorks Inc., Natick, MA, USA) where they were processed using trimmean, removing $30 \%$ of outliers. For this dataset, this means that from a total of 5642 points, 1693 outliers were removed and the remaining points were averaged. Using this process it was possible to reach an average value of 0.4001 . Like with Young's modulus, this value was also rounded, to 0.4 . This result is very close to the Poisson ratio of most materials commonly used for this type of blades, such as ABS's 0.4 [20].

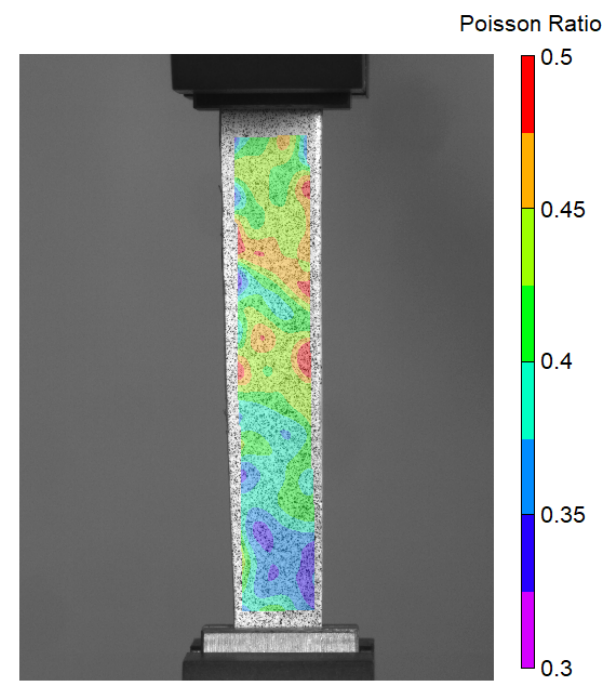

Figure 16. Poisson ratio results for the blade specimen.

\subsection{Model}

In the RC helicopter, two of these blades are assembled into a hub component and connected using pins. This connection has several degrees of freedom, namely the rotation of the blade relative to the hub, as well as some small gaps between the different parts.

The computational model was defined with three different domains, as shown in Figure 17: a solid domain and two fluid domains. In this approach, the solid part is considered static and a rotation speed is prescribed for the air in one fluid domain, resulting in similar conditions as if the blades themselves were rotating, as is commonly used in the literature [21]. This type of approach has a significant advantage when compared to simulating a moving solid and static fluid in its increased convergence. As an example, in our very first tests a moving solid solution was attempted, but it never converged. Nonetheless, the only major difference between these two situations is the lack of centrifugal force in the rotating fluid situation. Thus, in order to obtain an accurate representation, the blades were subjected to a prescribed centrifugal force, in addition to the forces caused by the air flow. 


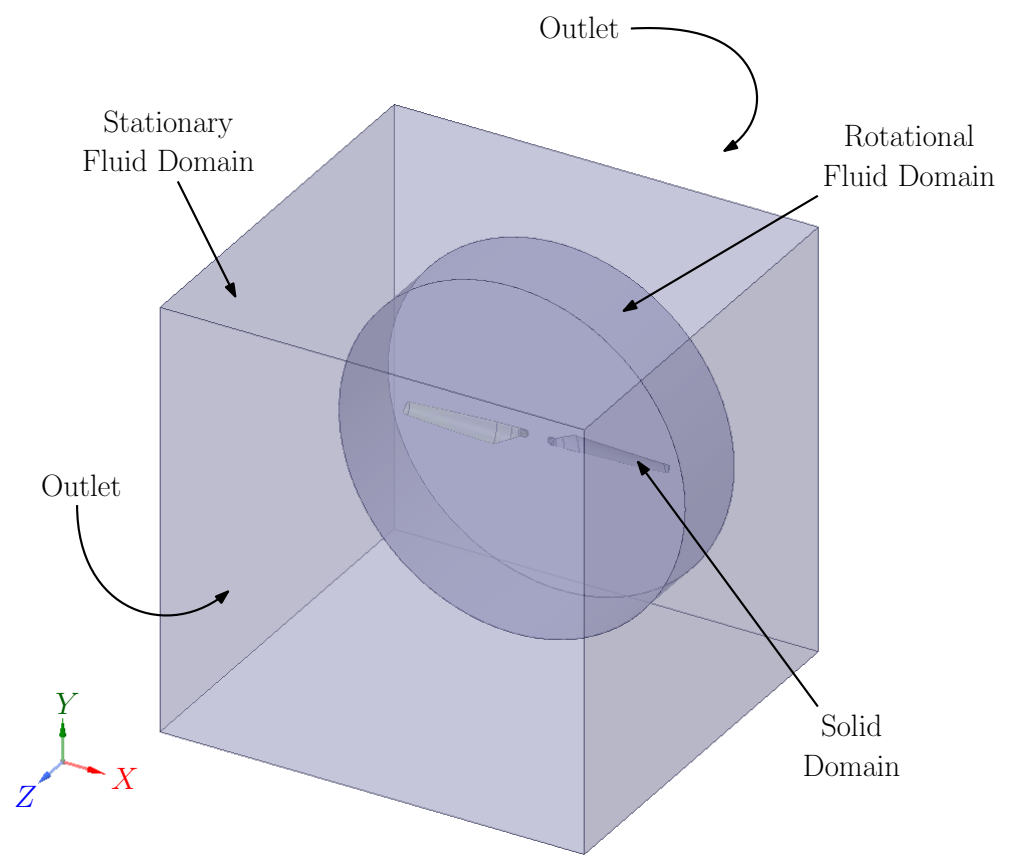

Figure 17. Boundary conditions and computational domains.

The two fluid domains are then one stationary and one rotational. The former includes the inlets/outlets from which air can enter or leave the computational domain and corresponds to the region where the air can be considered to be static, interfacing with the latter through a surface. The rotational domain is the one with an applied rotation speed, defined as $680 \mathrm{rpm}$ for these models, which was the measured speed in the associated DIC experiment.

The third domain, the solid one, consists of the rotating blades. The two blades were considered fixed in space, through a fixed support on their connection hole's inner surface, at the distance they would be if connected to the hub.

The origin of the coordinate system belongs to the rotation axis and to the blades' plane. Additionally, the coordinate system $(X-Y-Z)$ was aligned with the ones that are obtained using the methodology in [12], i.e., the rotation axis is along $Z, X$ is aligned with the left side of the blade's bottom edge, and $Y$ is perpendicular to both.

This solid domain was implemented in a transient FEA model, using the obtained material properties and the previously mentioned loads, i.e., the centrifugal and fluid-solid interface ones, and the prescribed supports.

The CFD component of the simulation, which includes the two fluid domains, uses air as its work fluid, modelled as an ideal gas with a density of $1.225 \mathrm{~kg} \mathrm{~m}^{-3}$ and a viscosity of $1.7894 \times 10^{-5} \mathrm{~kg} \mathrm{~m}^{-1} \mathrm{~s}^{-1}$.

It employs a pressure-based solver, in double-precision mode and with absolute velocity formulation, and the SIMPLE pressure-velocity coupling algorithm [22,23]. Turbulence is modelled using Reynolds stress model, with linear pressure-strain, scalable wall functions and wall reflection effects. Its parameters are shown in Table 1, where the first two relate to the dissipation rate, the next four to the linear pressure-strain model and the last to turbulent viscosity.

Like FEA, this is also a transient simulation. In this case, with second order implicit formulation.

Finally, the fluid-solid interface is modelled as a dynamic mesh, which will move according to the displacements calculated in the FEA model.

The coupled simulation was then ran with time increments of $0.01 \mathrm{~s}$ for a total of $0.5 \mathrm{~s}$, which corresponds to the time necessary for the results to stabilize. 
Table 1. Parameter values used for turbulence definition using Reynolds stress model.

\begin{tabular}{cc}
\hline Parameter & Value \\
\hline$C_{\varepsilon_{1}}$ & 1.44 \\
$C_{\varepsilon_{2}}$ & 1.92 \\
$C_{1}$ & 1.8 \\
$C_{2}$ & 0.6 \\
$C_{1}^{\prime}$ & 0.5 \\
$C_{2}^{\prime}$ & 0.3 \\
$C_{\mu}$ & 0.09 \\
\hline
\end{tabular}

\section{Results and Discussion}

In the initial versions of the model, such as the one in [13], there was a significant disagreement between the numerical and the experimental results. These were due to four main factors:

- The initial models used an inaccurate 3D shape obtained from a single image acquisition from the DAVID structured light scanner, without the use of dry powder;

- The Young's modulus was obtained using a method that was dependent on the inaccurate 3D shape, which in turn resulted in a wrong result;

- The existence of unmodelled gaps between the blades and the hub, which allowed for free rotation of the blades;

- Instability of the rotation axis, as it deformed due to its extended use.

The first two sources of error were already approached in previous sections.

The measured gaps are discussed in [13], and could permit rotations of up to $1.66^{\circ}$ perpendicular to $Z$, which could translate to a tip Z-displacement of up to $4.5 \mathrm{~mm}$. To correct this issue, the gaps were closed as well as possible, tightening the connection between the hub and the blade.

Although the influence of the gaps was smaller than those $4.5 \mathrm{~mm}$, it still had a large contribution, as can be seen in Figure 18 where the rotating blade's deformation is visually compared with and without gaps at the same speed.

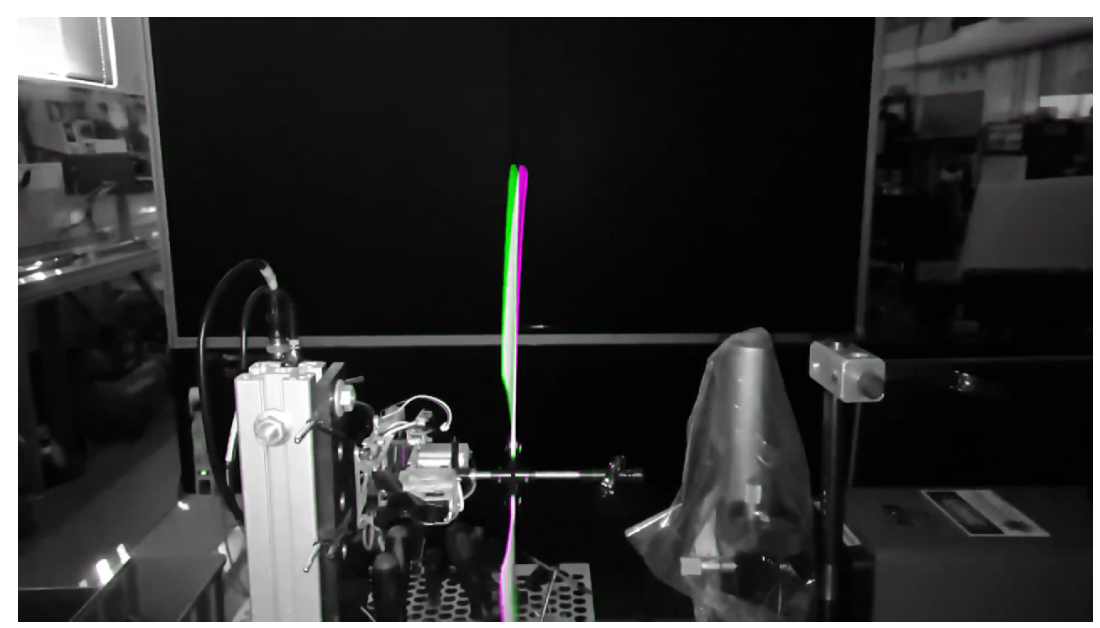

Figure 18. Comparison of the blades' rotating position before (purple) and after (green) reducing gaps.

The final source of error relates to an instability in the rotation axis, where it would cause vibration as it bends slightly. This was corrected by reducing the distance between the blades' hub and the helicopter's body and adding a support on the far side of the beam, implemented using a bearing and a teflon support, as shown in Figure 19. 


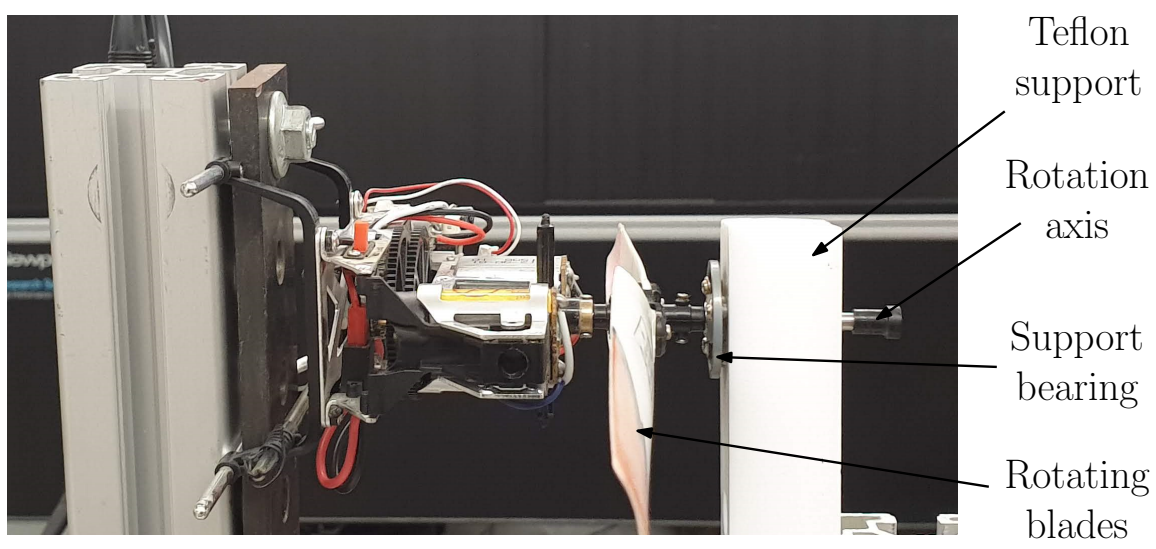

Figure 19. Stabilization of the RC helicopter's axis, using a bearing and a support built out of teflon.

For the present work, the objective centres around the structure's response and not the fluid's. Nonetheless, the air velocity in stationary frame at the final instant of the CFD simulation is shown in Figure 20 as an example.
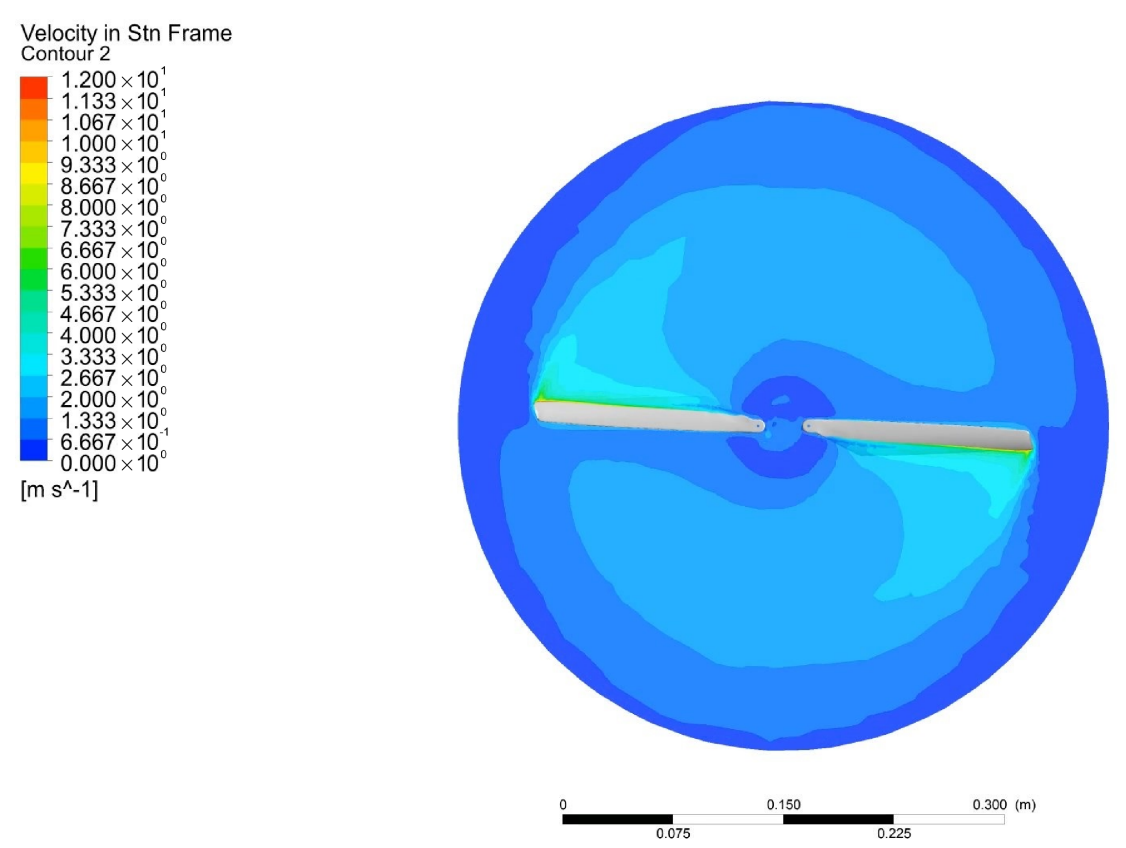

Figure 20. Fluid simulation result—velocity in stationary frame at the $0.5 \mathrm{~s}$ instant.

Comparably, among the structure's response parameters, the most important one is the displacement along the $Z$ direction, as it is the largest and thus the most significant one. Figure 21 shows a representation of these displacements overlaid on the obtained deformed shape of the blades, where the dominance of the displacements along $Z$ is visible.

In order to have comparable experimental results, it is necessary to apply the correction method described in [12] to the results of digital image correlation. That allows the use of coherent coordinate systems in both, which would not be the case otherwise. 

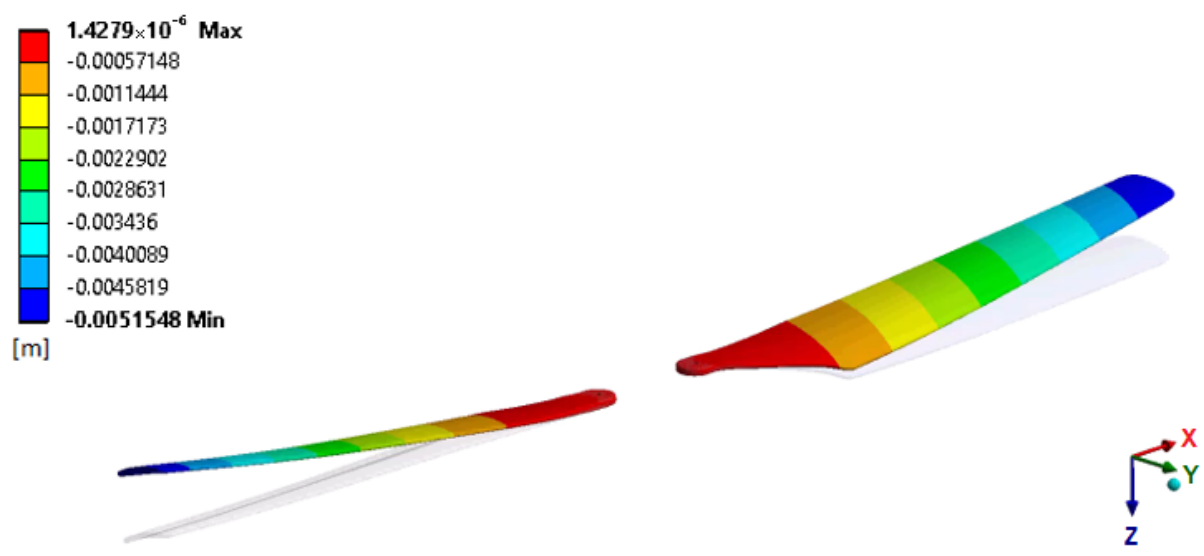

Figure 21. Mechanical simulation result-vdisplacement in $Z$ direction at the $0.5 \mathrm{~s}$ instant.

With this, it is possible to compare the obtained displacements. Regarding the experimental results, a particular frame was selected as the comparison point. Nonetheless, they were all very similar, presenting only some slight fluctuations, as is to be expected.

Figure 22 shows the results along the $X$ direction both in the numeric simulation (Figure 22a) and the experiment (Figure 22b). The considered coordinate systems are shown in each case. In the experimental one, it is very clear that there is a significant difference between the corrected coordinate system and the original one (with horizontal $X$ and vertical $Y$ ).

The displacements themselves present a very similar distribution, with very close values. It should be noted, however, that the numeric result's legend includes negative values, which are due to the other blade that is not shown here.
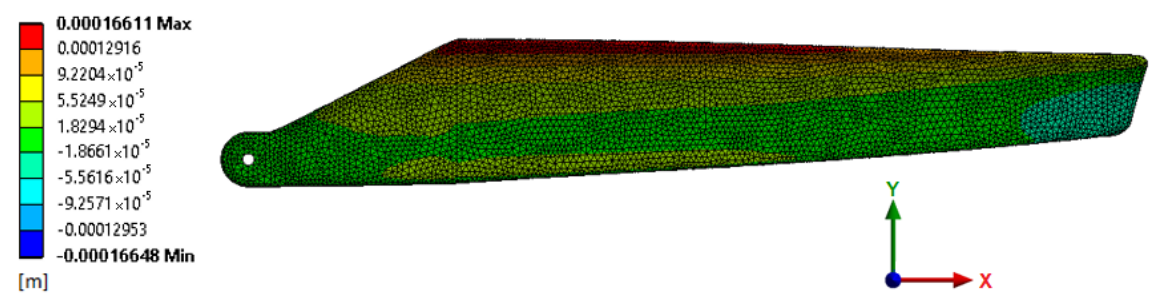

(a) Numeric

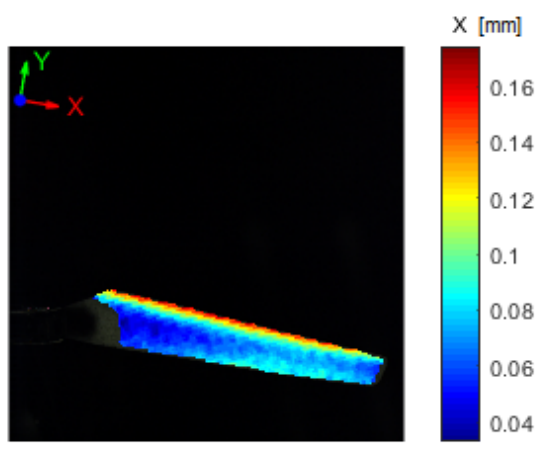

(b) Experimental

Figure 22. Obtained displacements along the $X$ direction.

The displacements along $Y$, shown in Figure 23a for the numeric case and in Figure 23b for the experimental one, also feature a very similar distribution. However, there is a very slight $(0.2 \mathrm{~mm})$ difference in magnitude. 

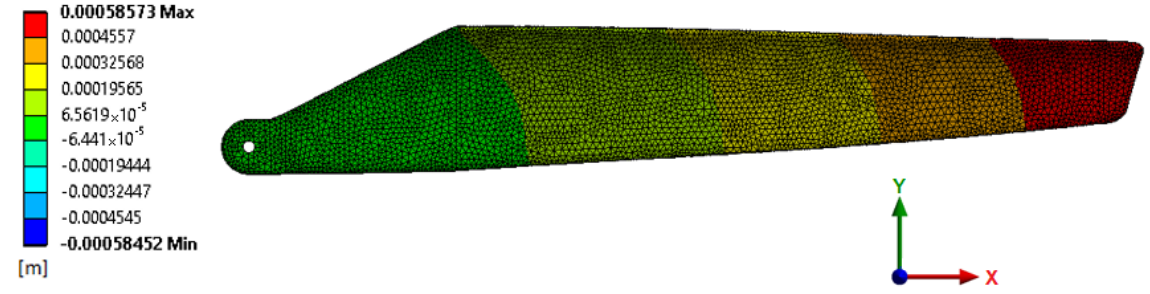

(a) Numeric

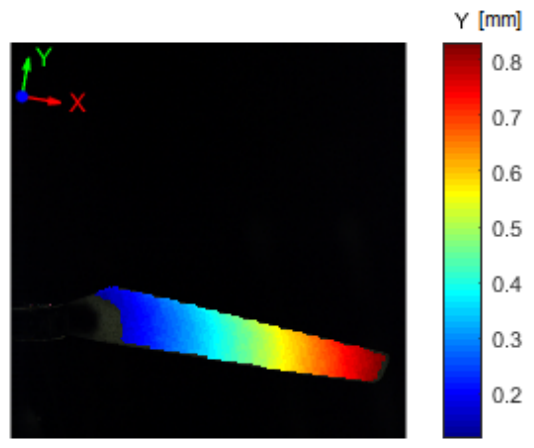

(b) Experimental

Figure 23. Obtained displacements along the $Y$ direction.

Finally, the $Z$ displacements are shown in Figure 24. Here, it is possible to see once again a very similar distribution with only slight difference in magnitude $(-5.15 \mathrm{~mm}$ vs. $-4.85 \mathrm{~mm})$. It should be noted that, due to some vibrating motions that are to be expected in a rotating object, these displacements fluctuate, which means that there were other frames closer to the $-5 \mathrm{~mm}$ mark.

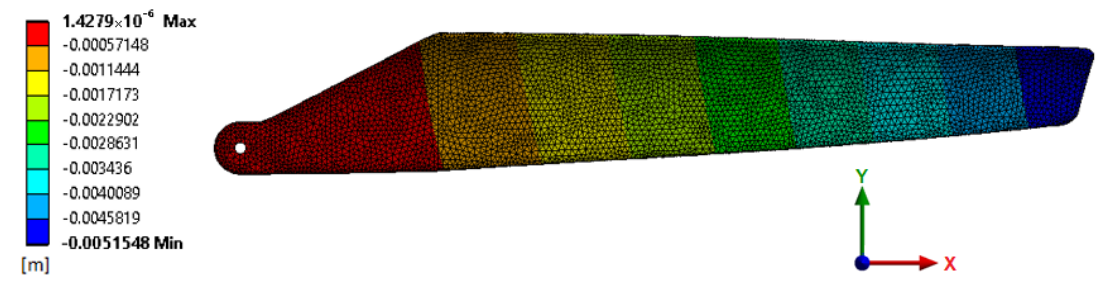

(a) Numeric

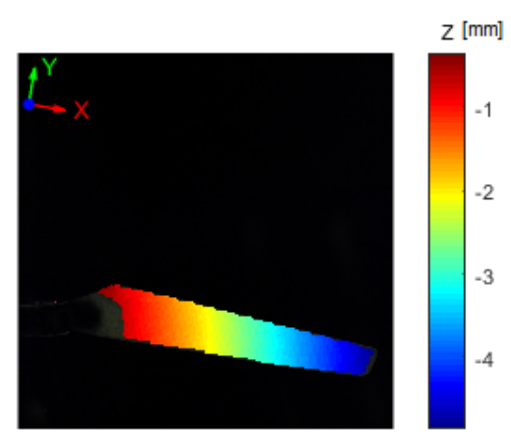

(b) Experimental

Figure 24. Obtained displacements along the $Z$ direction.

The total displacement for a chord that passes approximately through the middle of the blade was extracted from both datasets, and is shown in Figure 25a. It is possible to notice that the largest absolute differences are near the tip, with a maximum of $0.2769 \mathrm{~mm}$. In percentage terms, the difference between 
the two situations is consistently around 5\%, as shown in Figure 25b. The exception to this is found around the $65 \mathrm{~mm}$ mark, where the largest relative differences are found, reaching $8 \%$. Nonetheless, in absolute terms, this relative difference still corresponds only to $0.0237 \mathrm{~mm}$.

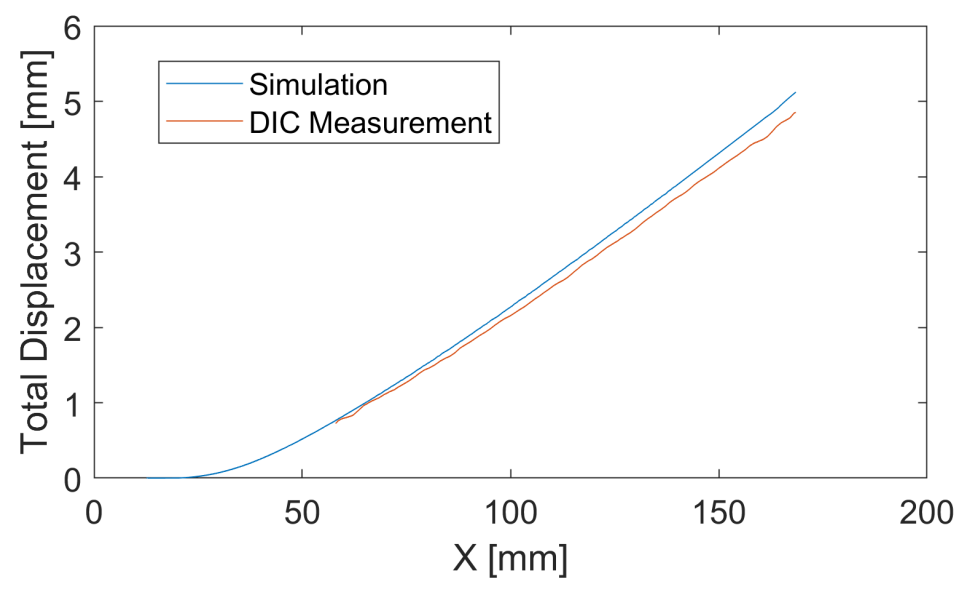

(a)

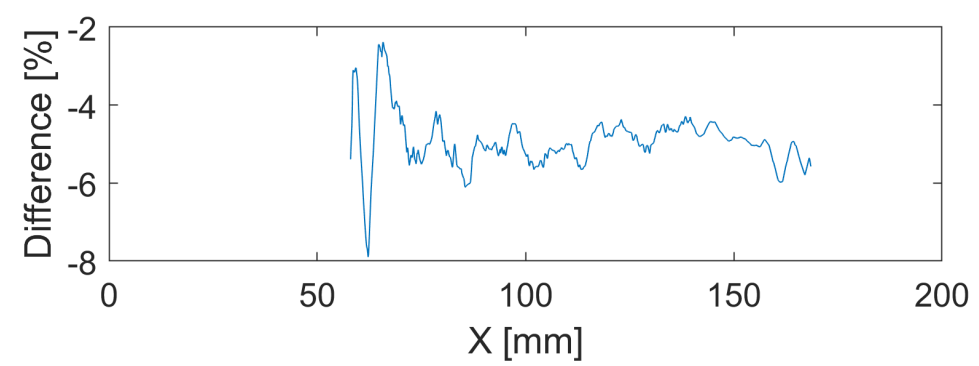

(b)

Figure 25. (a) Comparison of the experimental and numeric results for the displacements along a blade chord; (b) Percentage difference between the two methods.

It should be noted that, for these plots, the DIC approach's results only start at around $X=58 \mathrm{~mm}$ because that is the leftmost position that is not obscured by the helicopter's body in the selected chord.

There are multiple reasons that could explain the slight differences, such as vibrations and material properties variations for example. Nevertheless, the experimental and numeric results are very close, with very similar distributions, which is an encouraging result.

\section{Conclusions}

The main objective for this work was to perform an experimental and numerical analysis of the deformation experienced by an RC helicopter's blades in operation. It was possible to achieve this, obtaining agreeing results in both.

Compared with the author's previous work [13], the presented experimental and numerical results are significantly more consistent, mainly due to corrections performed not only experimentally, such as the removal of gaps between the blades and the hub, but also in the problem definition phase, such as the improvement of the acquired shape and the use of a destructive method to obtain material properties.

Additionally, the differences between this work and [13] also highlight the importance of validation in computational models, as small differences between the simulation and the real case may have a significant impact on the results.

It was possible to obtain agreeing results when comparing absolute displacements, not only in the out-of-plane direction but also in-plane ones. This complements the work of Winstroth et al. [6], 
where the comparison of absolute deformations, both out-of-plane and in-plane, is listed among the real challenges for application of similar methods. In the present work, all analyses were performed considering absolute displacements.

These results help to showcase the developed method for experimental measurement of displacements in rotating objects, as well as the importance of the data correction methodology presented in [12]. That procedure is responsible for reconciling the experimental and numeric coordinate systems and properly aligning the obtained data. Without this, it would not be possible to compare absolute and in-place displacements in this work.

The main limitation of the experimental approach is the amount of light that is necessary to acquire clear images of a rotating object, particularly with high speeds. In those cases, this may require multiple light sources which at some point may become difficult to manage. Another limitation is related to the triggering system. For each event, i.e., every time it counts $m$ pulses, it is only capable of outputting one pulse per channel. That is, it does not support time-based triggering in addition to the position-based one. This factor is not important when using high-speed cameras, which can provide their own time-based image acquisition after an external trigger, but may be significant for the use of industrial cameras, which cannot do the same.

Regarding the numeric approach, the main limitation lies in the necessary time to obtain a solution. This particular model took $5 \mathrm{~h}$ to solve with less than 500k CFD cells and 100k FEA elements. The usage of a complex turbulence model such as the Reynolds stress model contributes to a longer solution time. Thus, it would be interesting in the future to analyse how other turbulence models fare regarding solution time and result agreement.

Additional future work in this area will look into the application of these methods to operational modal analysis and other vibration analyses. The use of time methods with impulse responses should be feasible, but the most interesting approach would be to analyse vibrations in normal operating behaviour, for example using random decrement techniques. Additionally, in order to further improve these results, an interesting next step would be re-include the hub into the simulation and analyse the influence of its presence.

Author Contributions: Conceptualization, P.J.S., F.B., P.J.T. and P.M.G.P.M.; methodology, P.J.S. and F.B.; software, P.J.S.; validation, P.J.S., P.J.T. and P.M.G.P.M.; investigation, P.J.S.; resources, P.J.T. and P.M.G.P.M.; writing-original draft preparation, P.J.S.; writing-review and editing, P.J.S., F.B., P.J.T. and P.M.G.P.M.; visualization, P.J.S.; supervision, P.J.T. and P.M.G.P.M.; project administration, P.M.G.P.M. All authors have read and agreed to the published version of the manuscript.

Funding: This reasearch was funded by Fundação para a Ciência e a Tecnologia under PhD scholarship SFRH/BD/129398/2017.

Conflicts of Interest: The authors declare no conflict of interest.

\section{Abbreviations}

The following abbreviations are used in this manuscript:

$\begin{array}{ll}\text { ABS } & \text { Acrylonitrile butadiene styrene } \\ \text { CFD } & \text { Computational Fluid Dynamics } \\ \text { DIC } & \text { Digital Image Correlation } \\ \text { FEA } & \text { Finite Element Analysis } \\ \text { fps } & \text { Frames per second } \\ \text { LED } & \text { Light Emitting Diode } \\ \text { MP } & \text { Megapixel } \\ \text { RC } & \text { Remote Controlled }\end{array}$




\section{References}

1. Bucher, I.; Ewins, D.J. Modal analysis and testing of rotating structures. Philos. Trans. R. Soc. Lond. Ser. A Math. Phys. Eng. Sci. 2001, 359, 61-96. [CrossRef]

2. Winstroth, J.; Seume, J.R. Wind Turbine Rotor Blade Monitoring using Digital Image Correlation: Assessment on a Scaled Model. In Proceedings of the 32nd ASME Wind Energy Symposium, National Harbor, MD, USA, 13-17 January 2014; American Institute of Aeronautics and Astronautics: Reston, VA, USA, 2014. [CrossRef]

3. Bazilevs, Y.; Hsu, M.C.; Kiendl, J.; Wüchner, R.; Bletzinger, K.U. 3D simulation of wind turbine rotors at full scale. Part II: Fluid-structure interaction modeling with composite blades. Int. J. Num. Methods Fluids 2011, 65, 236-253. [CrossRef]

4. Santo, G.; Peeters, M.; Van Paepegem, W.; Degroote, J. Dynamic load and stress analysis of a large horizontal axis wind turbine using full scale fluid-structure interaction simulation. Renew. Energy 2019. [CrossRef]

5. Stürmer, A.; Marquez Gutierrez, C.O.; Roosenboom, M.E.W.; Schröder, A.; Geisler, R.; Pallek, D.; Agoc, J.; Neitzke, K.P. Experimental and Numerical Investigation of a Contra Rotating Open-Rotor Flowfield. J. Aircraft 2012, 49, 1868-1877. [CrossRef]

6. Winstroth, J.; Schoen, L.; Ernst, B.; Seume, J.R. Wind turbine rotor blade monitoring using digital image correlation: A comparison to aeroelastic simulations of a multi-megawatt wind turbine. J. Phys. Conf. Ser. 2014, 524, 12064. [CrossRef]

7. Winstroth, J.; Seume, J.R. Wind turbine rotor blade monitoring using digital image correlation: $3 \mathrm{~d}$ simulation of the experimental setup. In Proceedings of the European Wind Energy Association-EWEA 2014, Barcelona, Spain, 10-13 March 2014.

8. Sirohi, J.; Lawson, M.S. Measurement of helicopter rotor blade deformation using digital image correlation. Opt. Eng. 2012, 51, 43603-43608. [CrossRef]

9. Boden, F.; Bodensiek, K.; Stasicki, B. Application of image pattern correlation for non-intrusive deformation measurements of fast rotating objects on aircrafts. Proc. SPIE Int. Soc. Opt. Eng. 2009, 7522, 75222S1-75222S10.

10. Sousa, P.J.; Barros, F.; Tavares, P.J.; Moreira, P.M. Displacement measurement and shape acquisition of an RC helicopter blade using Digital Image Correlation. Proc. Struct. Integr. 2017, 5, 1253-1259. [CrossRef]

11. Sousa, P.J.; Barros, F.; Tavares, P.J.; Moreira, P.M. Digital image correlation displacement measurement of a rotating RC helicopter blade. Eng. Fail. Anal. 2018, 90, 371-379. [CrossRef]

12. Sousa, P.J.; Tavares, J.M.R.; Tavares, P.J.; Moreira, P.M. Correction of rigid body motion in deformation measurement of rotating objects. Measurement 2018, 129, 436-444. [CrossRef]

13. Sousa, P.J.; Barros, F.; Tavares, P.J.; Moreira, P.M. Displacement analysis of rotating RC helicopter blade using coupled CFD-FEA simulation and digital image correlation. Proc. Struct. Integr. 2019, 17, 812-821. [CrossRef]

14. Sousa, P.J.; Carneiro, F.; Ramos, N.V.; Tavares, P.J.; Moreira, P.M. Development of LED-based illumination system for high-speed digital image correlation. Proc. Struct. Integr. 2019, 17, 828-834. [CrossRef]

15. Thorlabs Inc. Photodiode Tutorial. Available online: https://www.thorlabs.com/tutorials.cfm?tabID= 787382FF-26EB-4A7E-B021-BF65C5BF164B (accessed on 30 January 2020).

16. AP Technologies Ltd. Photodiode Theory of Operation. Available online: https://www.aptechnologies.co. uk/support/SiPDs/operation (accessed on 30 January 2020).

17. OSI Optoelectronics. Photoconductive Series - Planar Diffused Silicon Photodiodes. Available online: http: / / www.osioptoelectronics.com/Libraries/Datasheets/Photoconductive-Photodiodes.sflb.ashx (accessed on 30 January 2020).

18. Texas Instruments Inc. THS4631-High-Voltage, High Slew Rate, Wideband Fet-Input Operational Amplifier. 2011. Available online: https:/ / www.ti.com/lit/ds/symlink/ths4631.pdf (accessed on 30 January 2020).

19. Cignoni, P.; Callieri, M.; Corsini, M.; Dellepiane, M.; Ganovelli, F.; Ranzuglia, G. MeshLab: An Open-Source Mesh Processing Tool. In Eurographics Italian Chapter Conference; Scarano, V., Chiara, R.D., Erra, U., Eds.; The Eurographics Association: Geneve, Switzerland, 2008. [CrossRef]

20. Granta Design Limited. ABS (High-Impact, Injection Molding), CES Edupack Software; Granta Design Limited: Cambridge, UK, 2019.

21. Chen, Y.B.; Wang, Z.K.; Tsai, G.C. Two-way Fluid-Structure Interaction Simulation of a Micro Horizontal Axis Wind Turbine. Int. J. Eng. Technol. Innov. 2015, 33-44. 
22. ANSYS Inc. ANSYS ${ }^{\circledR}$ Fluent, Release 18.2, Help System, Theory Guide-21.4.3. Pressure-Velocity Coupling; ANSYS Inc.: Canonsburg, PA, USA, 2017.

23. Patankar, S.V.; Spalding, D.B. A calculation procedure for heat, mass and momentum transfer in three-dimensional parabolic flows. Int. J. Heat Mass Trans. 1972, 15, 1787-1806. [CrossRef]

(C) 2020 by the authors. Licensee MDPI, Basel, Switzerland. This article is an open access article distributed under the terms and conditions of the Creative Commons Attribution (CC BY-NC-ND) license (https://creativecommons.org/licenses/by-nc-nd/4.0/). 